Supporting Information

\title{
Thermally Stable Passivation toward High Efficiency Inverted Perovskite Solar Cells
}

Robert D. J. Oliver, Yen-Hung Lin, Alexander J. Horn, Chelsea Q. Xia, Jonathan H. Warby, Michael B. Johnston, Alexandra J. Ramadan*, Henry J. Snaith*

Department of Physics, University of Oxford, Clarendon Laboratory, Parks Road, Oxford, OX1 3PU, UK

E-mail: Alexandra.ramadan@physics.ox.ac.uk, henry.snaith@physics.ox.ac.uk 


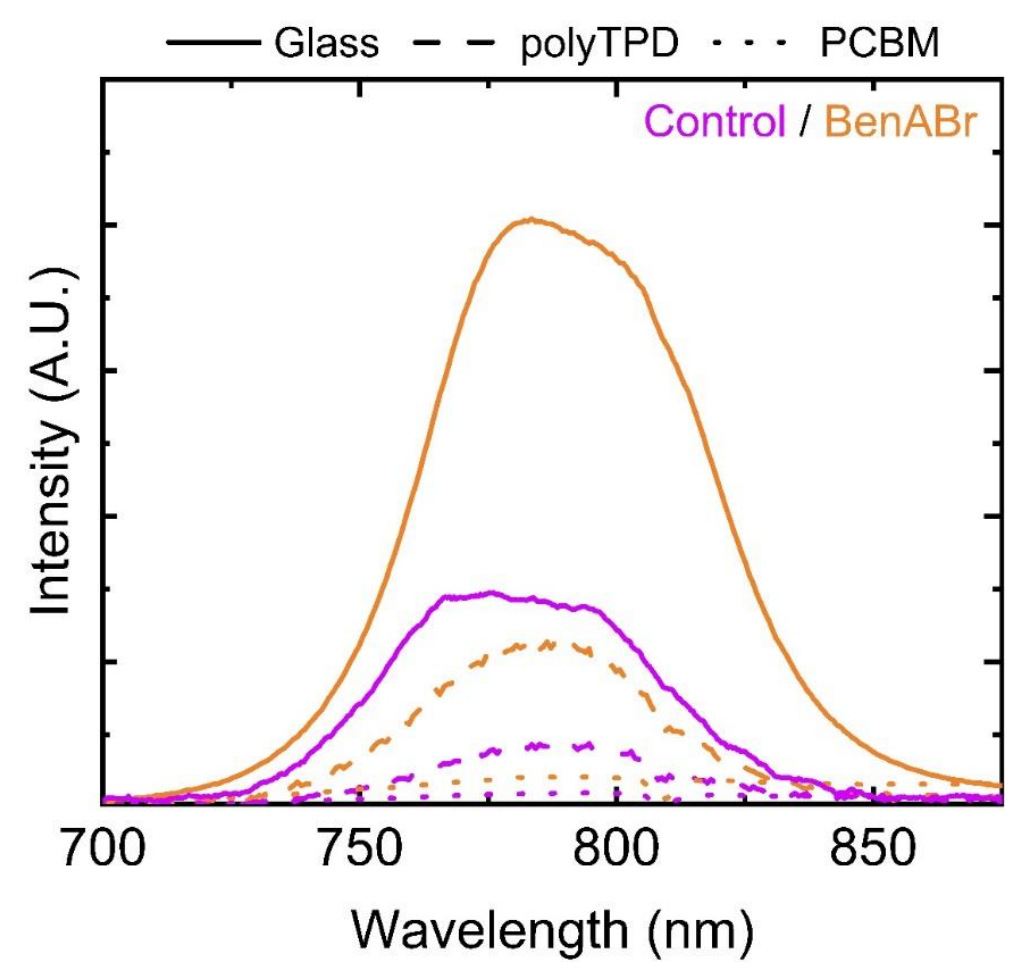

Figure S1. Photoluminescence spectra for treated (orange) and control (purple) samples on glass substrates (solid lines), isolated p-type contact (polyTPD, dashed lines) and n-type contact (PCBM, dotted lines). 


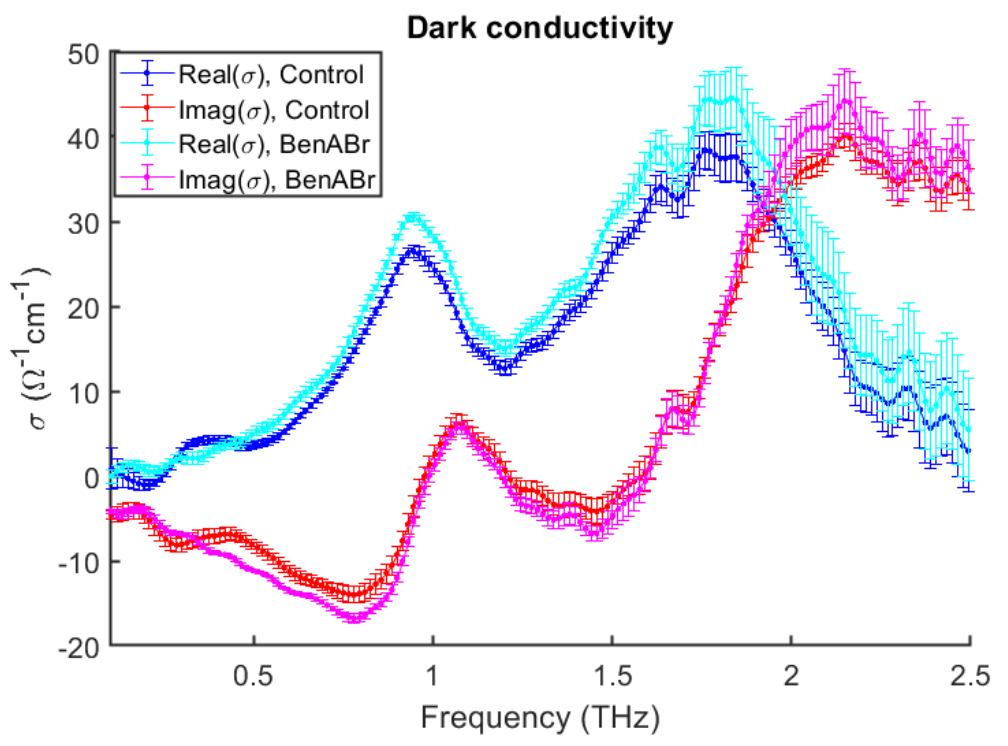

Figure S2. Dark conductivity spectra measured without photoexcitation. 


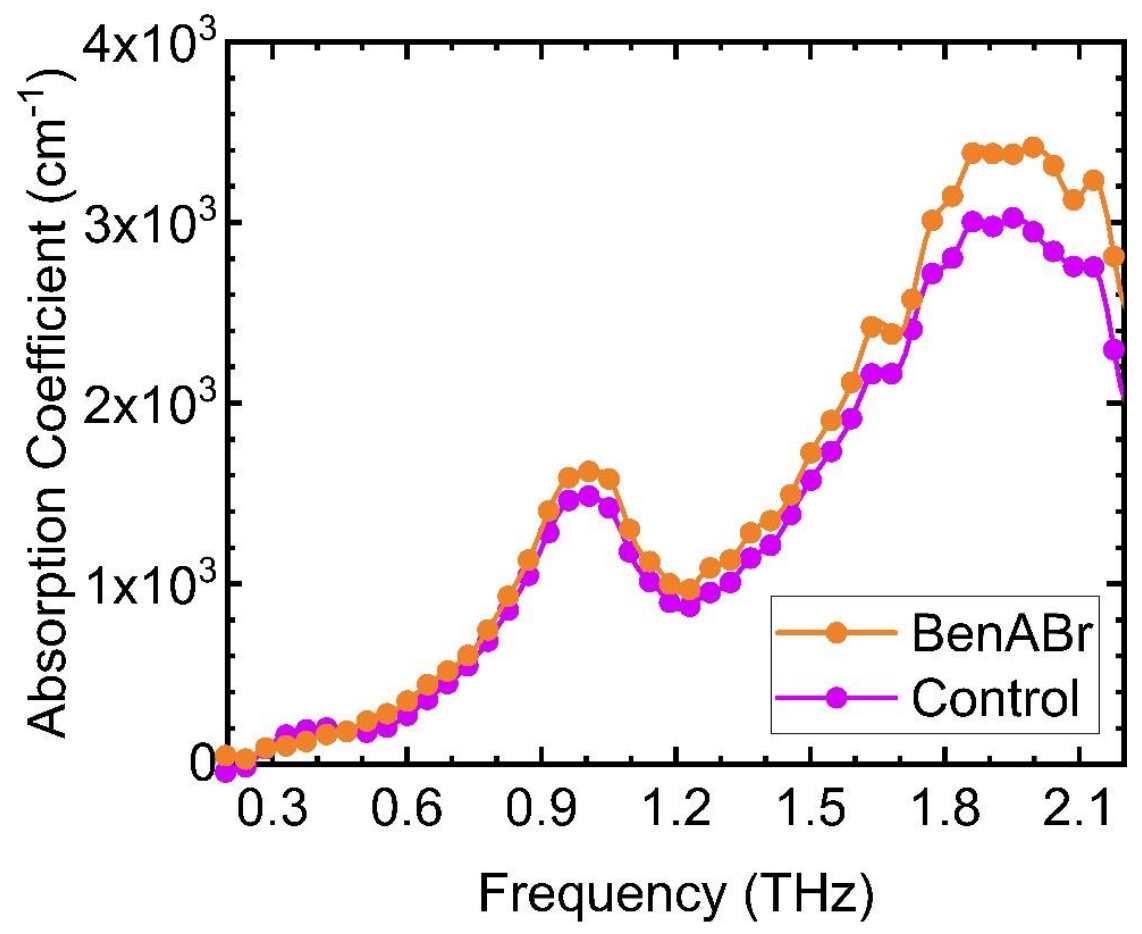

Figure S3. Absorption coefficient for pristine (purple) and treated (orange) films calculated from the dark conductivity presented in Figure S2. Full details of the calculation are presented in Supporting Note 3. 


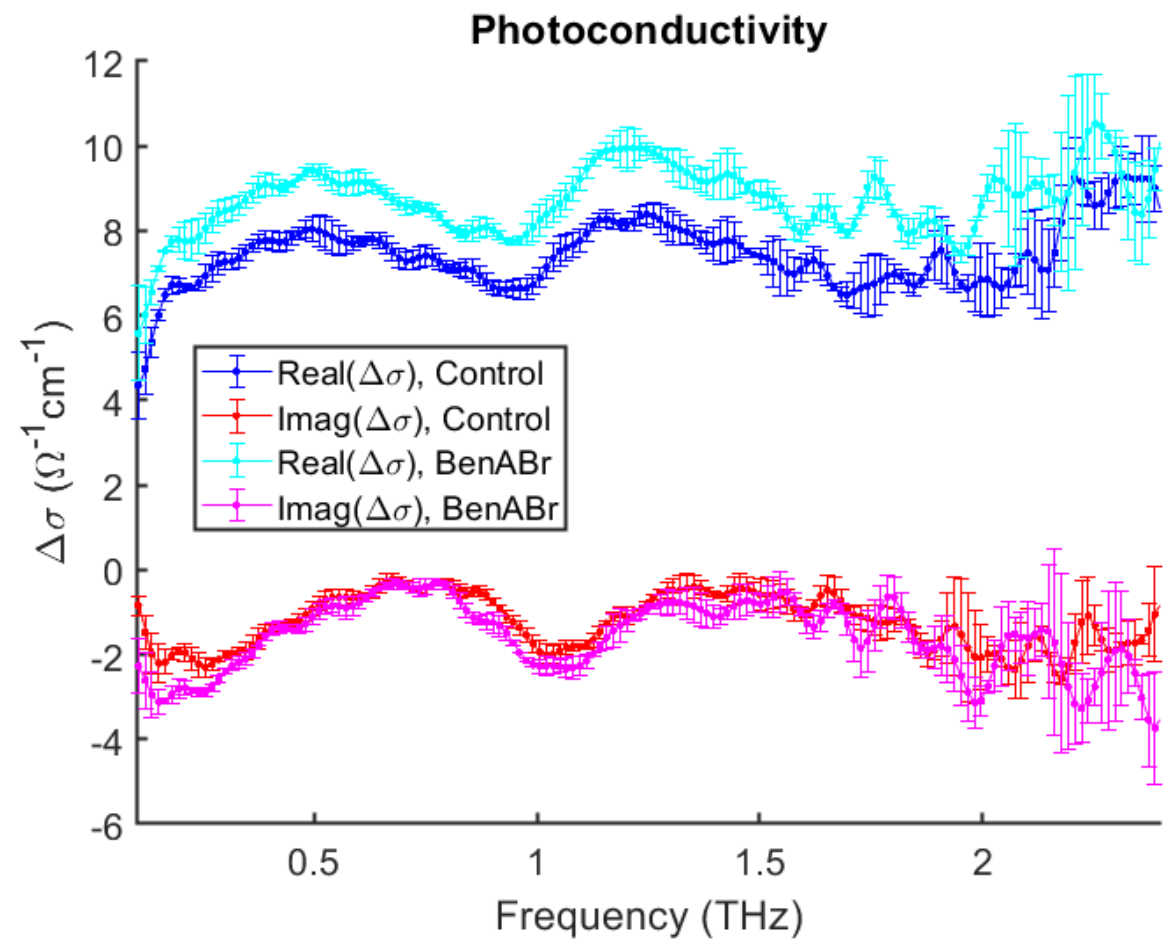

Figure S4. Photoconductivity spectra measured under photoexcitation by $400 \mathrm{~nm}$ wavelength $35 \mathrm{fs}$ laser pulses at fluence $5.2 \mu \mathrm{Jcm}^{-2}$. 

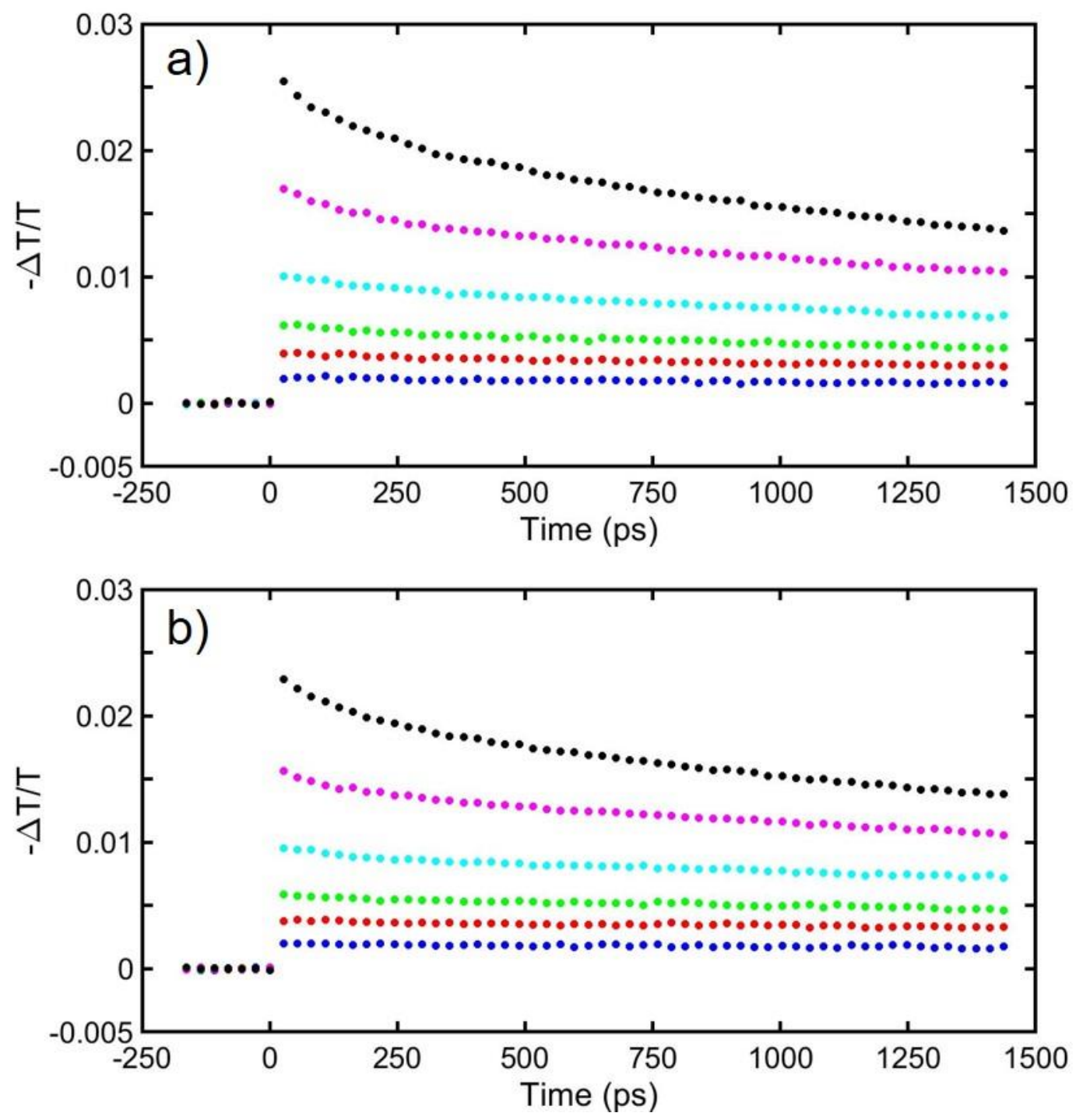

Figure S5. Photoinduced terahertz transients for a) control and b) treated films. The fluence range for these measurements was 1.2 (blue data) -13.5 (black data) $\mu \mathrm{J} \mathrm{cm}^{-2}$. 


\begin{tabular}{|c|c|c|}
\hline Element & Peak Position (eV) & $\begin{array}{ll}\text { Attributed } & \text { Bonding } \\
\text { Environments } & \end{array}$ \\
\hline \multicolumn{3}{|c|}{ Control film } \\
\hline $\operatorname{Br} 3 d$ & $68.8 / 69.8\left(\mathrm{Br} 3 d_{5 / 2} / \mathrm{Br} 3 d_{3 / 2}\right)$ & $\mathrm{Br}^{-}$ \\
\hline Cs $3 d$ & $725.2 / 739.2\left(\mathrm{Cs} 3 d_{5 / 2} / \mathrm{Cs} 3 d_{3 / 2}\right)$ & $\mathrm{Cs}^{+}$ \\
\hline $\mathrm{I} 3 d$ & 619.6/631.0 (I $3 d_{5 / 2} /$ I $\left.3 d_{3 / 2}\right)$ & $\mathrm{I}^{-}$ \\
\hline $\mathrm{C} 1 s$ & $285.1 / 286.8 / 288.6 / 289.2(\mathrm{C} 1 s)$ & $\mathrm{C}-\mathrm{C}, \mathrm{C}-\mathrm{H} / \mathrm{C}-\mathrm{O}-\mathrm{C} / \mathrm{O}-\mathrm{C}=\mathrm{O} / \mathrm{C}=\mathrm{O}$ \\
\hline $\mathrm{Pb} 4 f$ & $\begin{array}{l}137.6 / 138.7 / 142.6 / 143.6 \\
\left(\mathrm{~Pb} 4 f_{7 / 2} / \mathrm{Pb} 4 f_{5 / 2}\right)\end{array}$ & $\mathrm{Pb}^{2+} / \mathrm{Pb}^{0}$ \\
\hline \multicolumn{3}{|c|}{ BenABr treated film } \\
\hline $\mathrm{Br} 3 d$ & $68.8 / 69.8\left(\mathrm{Br} 3 d_{5 / 2} / \mathrm{Br} 3 d_{3 / 2}\right)$ & $\mathrm{Br}^{-}$ \\
\hline Cs $3 d$ & $725.2 / 739.2\left(\mathrm{Cs} 3 d_{5 / 2} / \mathrm{Cs} 3 d_{3 / 2}\right)$ & $\mathrm{Cs}^{+}$ \\
\hline $\mathrm{I} 3 d$ & 619.6/631.0 (I $3 d_{5 / 2} /$ I $\left.3 d_{3 / 2}\right)$ & $\mathrm{I}^{-}$ \\
\hline $\mathrm{C} 1 s$ & $284.1 / 285.2 / 286.8 / 288.6 / 289.2(\mathrm{C} 1 s)$ & $\begin{array}{l}\text { Aryl C/C-C, C-H/C-O-C/O- } \\
\mathrm{C}=\mathrm{O} / \mathrm{C}=\mathrm{O}\end{array}$ \\
\hline $\mathrm{Pb} 4 f$ & $\begin{array}{l}137.6 / 138.7 / 142.6 / 143.5 \\
\left(\mathrm{~Pb} 4 f_{7 / 2} / \mathrm{Pb} 4 f_{5 / 2}\right)\end{array}$ & $\mathrm{Pb}^{2+} / \mathrm{Pb}^{0}$ \\
\hline
\end{tabular}

Table S1. Peak positions of high resolution X-ray photoemission spectroscopy data. ${ }^{1}$ 

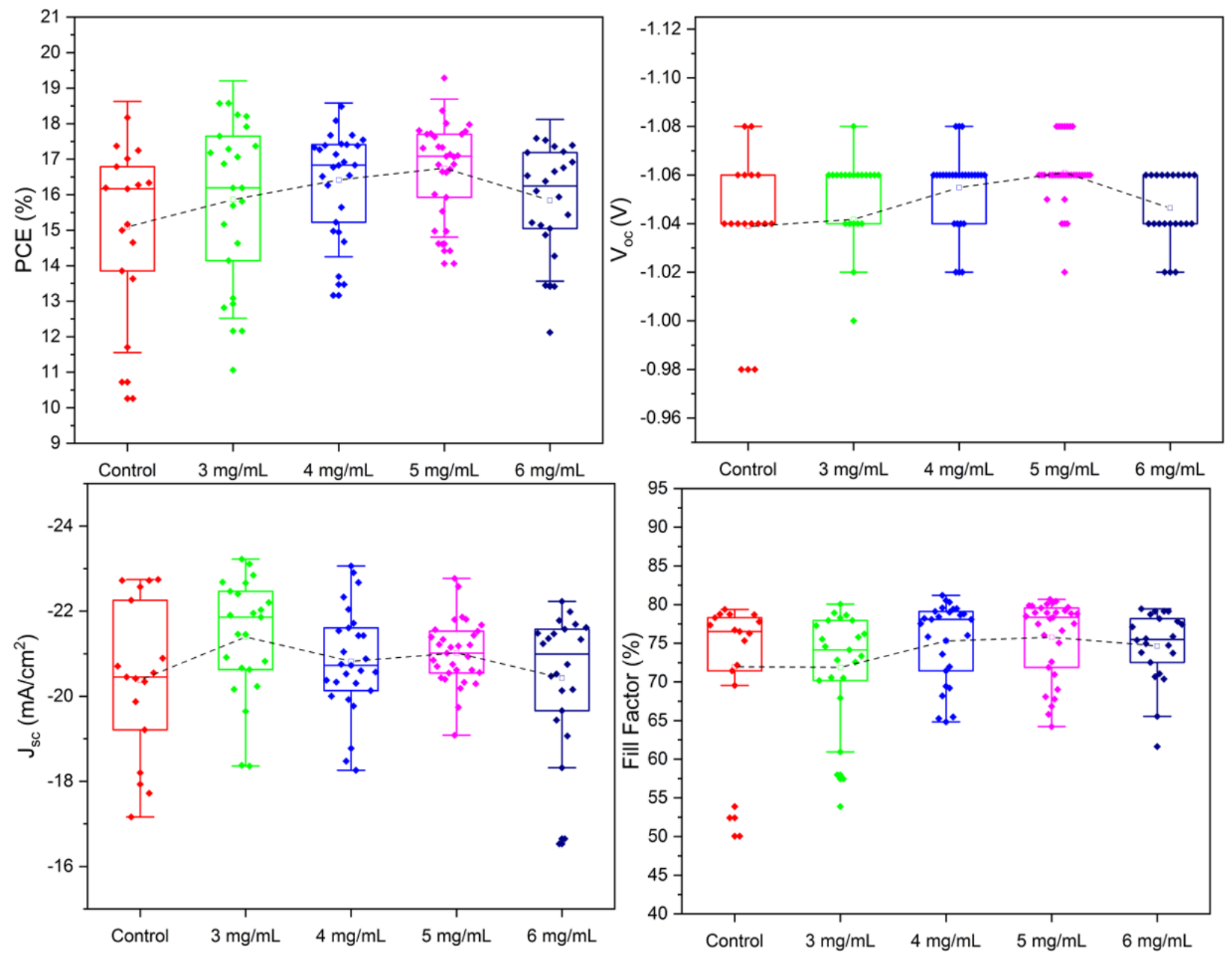

Figure S6. Statistical summary of the main device parameters for the concentration optimization for the Ben $\mathrm{ABr}$ passivation treatment. Dashed black lines connect the mean values of the different concentrations and are included as a guide to the eye. 

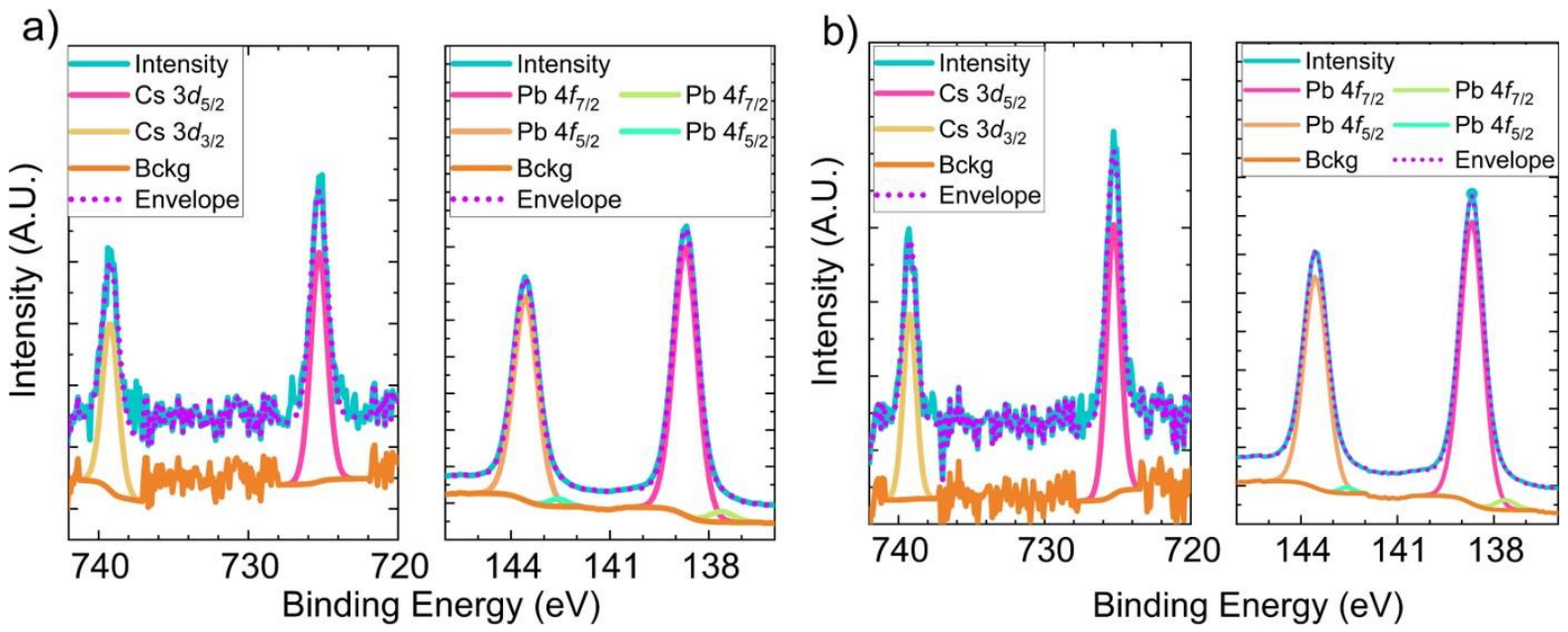

Figure S7. XPS fittings for the $\mathrm{Cs} 3 d$ and $\mathrm{Pb} 4 f$ core levels for a) pristine and $\mathrm{b}$ ) treated samples. The intensity and envelope have been offset to make the fittings clearer. Full details of peak positions can be found in Table $\mathbf{S 1}$.
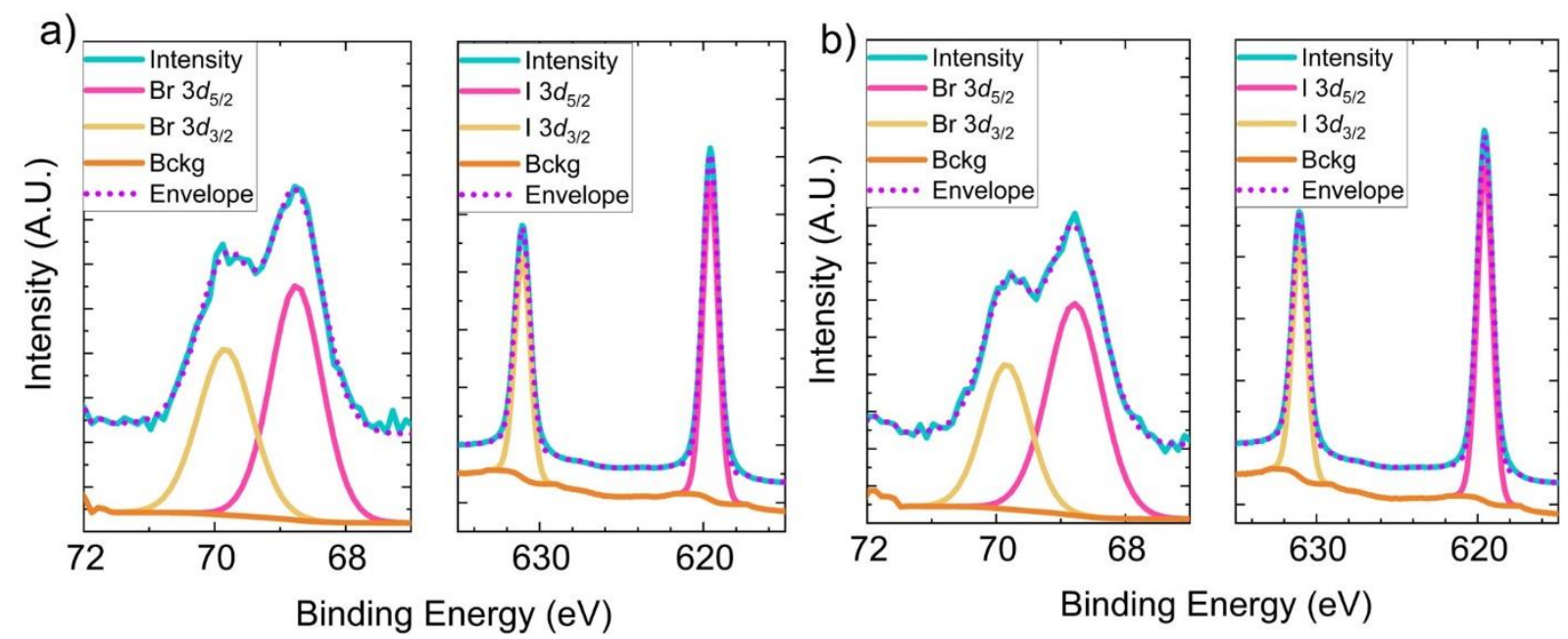

Figure S8. XPS fittings for the $\mathrm{Br} 3 d$ and I $3 d$ core levels for a) pristine and $\mathrm{b}$ ) treated samples. The intensity and envelope have been offset to make the fittings clearer. Full details of peak positions can be found in Table $\mathbf{S 1}$. 

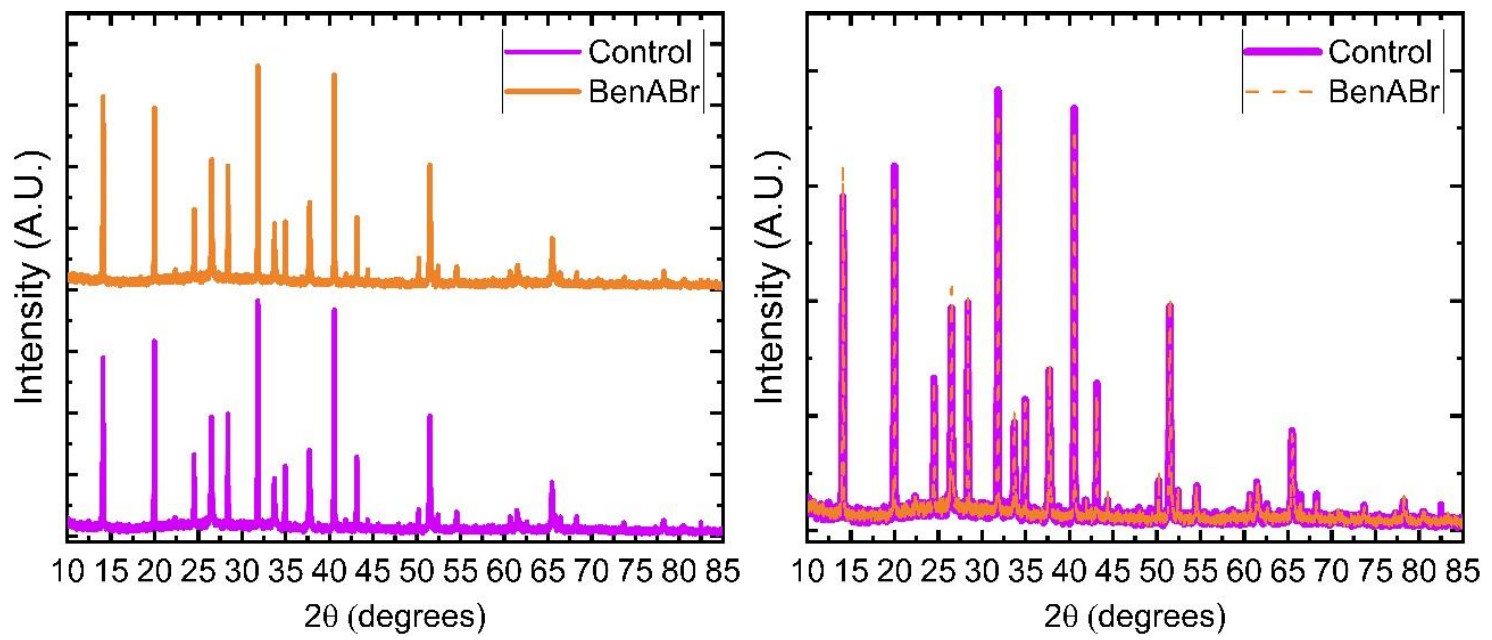

Figure S9. X-ray diffraction patterns for control (purple) and treated (orange) films on FTO substrates. The same pattern is shown offset (left) and overlapping (right). X-rays were generated by copper $\mathrm{K} \alpha \mathrm{X}$-ray source, $\lambda=0.1541 \mathrm{~nm}$
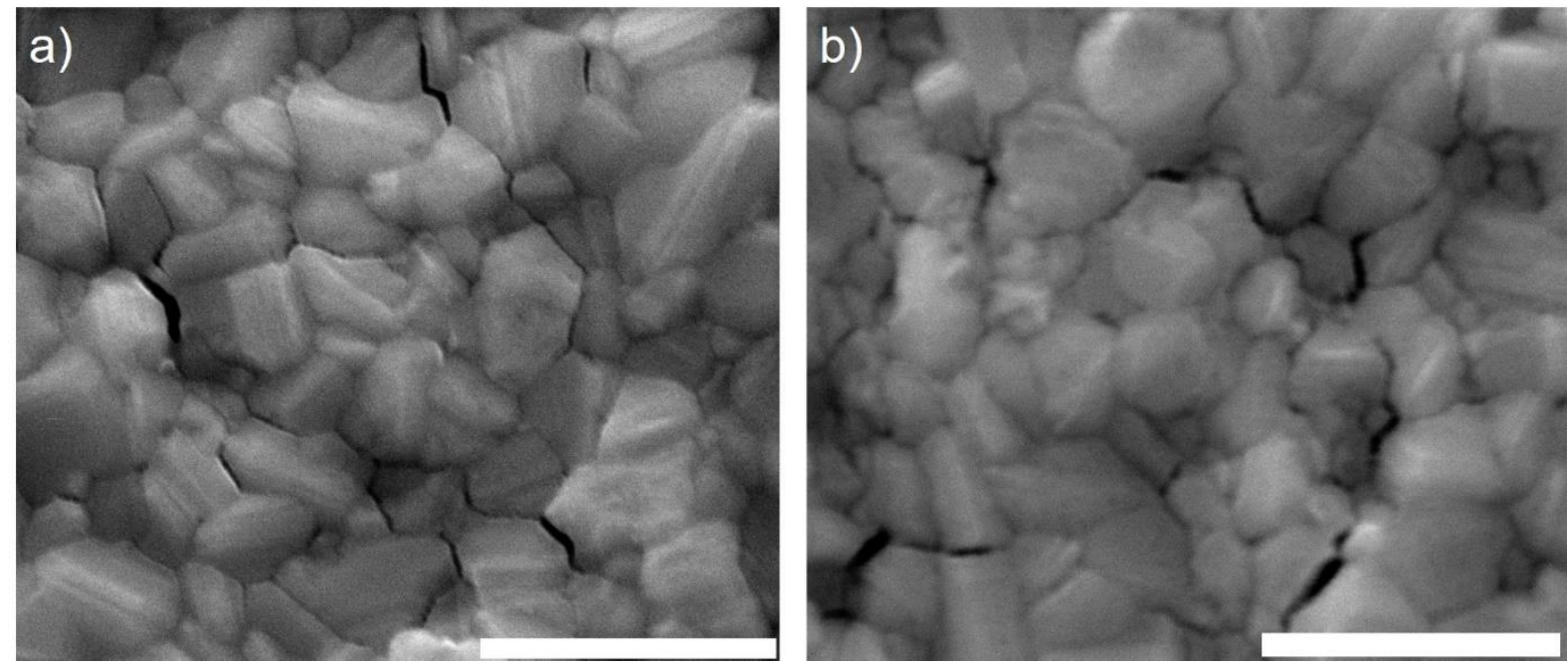

Figure S10. Scanning electron micrographs for a) control and b) treated samples. Scales bars are $1 \mu \mathrm{m}$. 


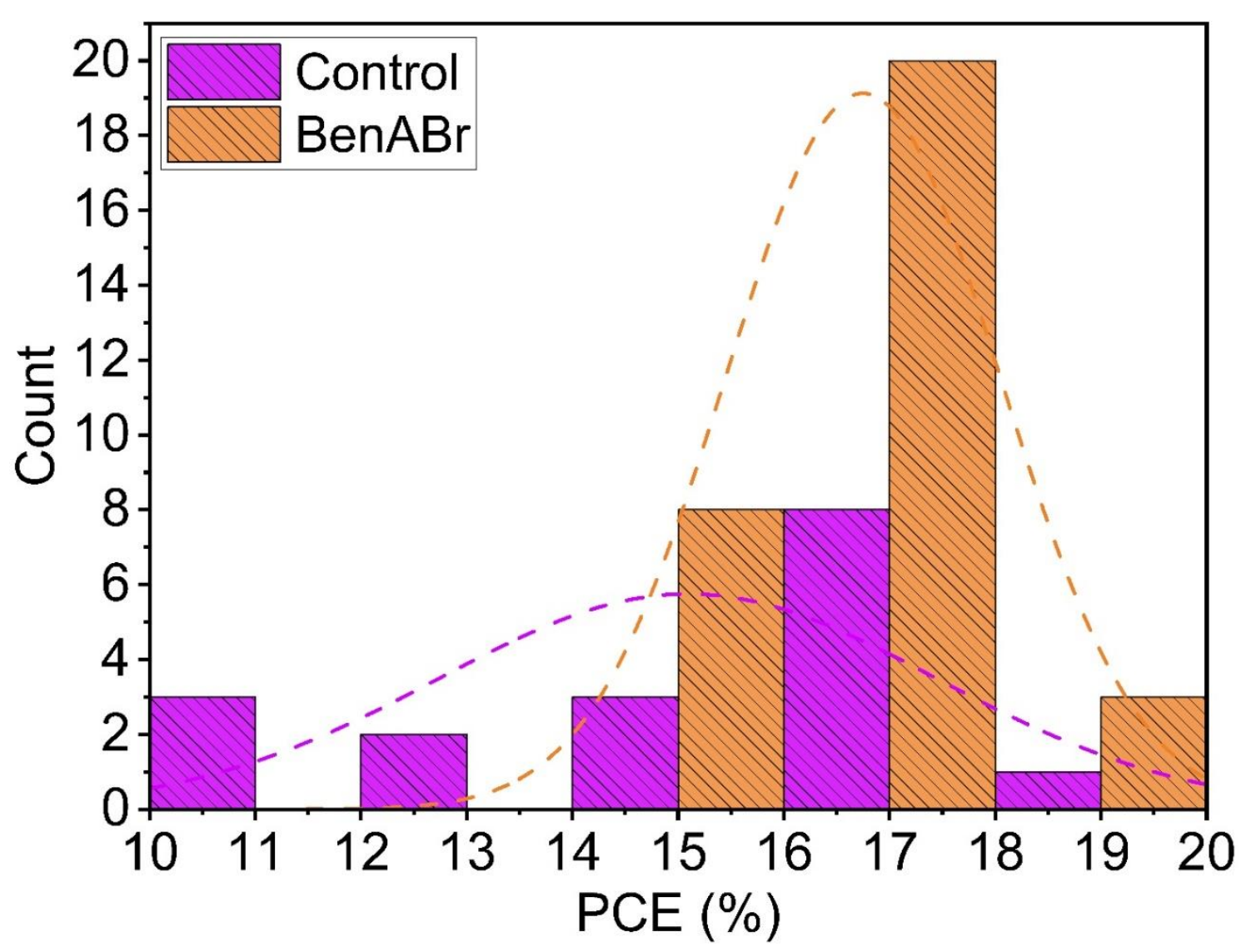

Figure S11. Histogram of the PCEs for control (purple) and treated (orange) devices. Dashed curves are normal distributions fitted to the data. 


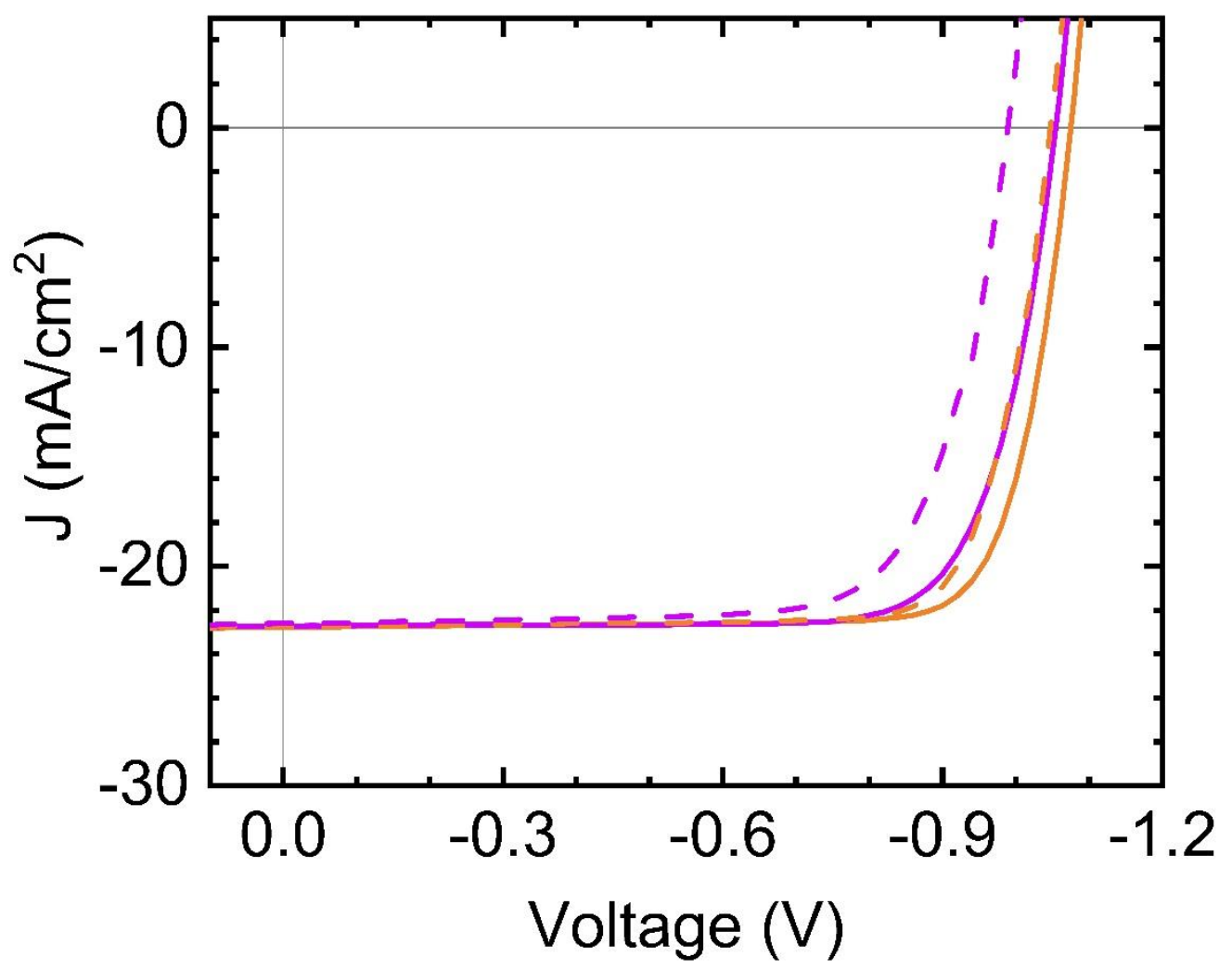

Figure S12. JV curves of the champion control (purple) and treated (orange) devices, forward (reverse) scans are shown in solid (dotted) lines. 


\section{Experimental Methods}

Benzylammonium bromide synthesis: $5 \mathrm{~mL}$ of benzylamine and $30 \mathrm{~mL}$ of ethanol were mixed and stirred in a $250 \mathrm{~mL}$ 2-neck flask in an ice-water bath. Then $7 \mathrm{~mL}$ of $\mathrm{HBr}$ acid $(48 \mathrm{wt} \%$ in water) was slowly added into the mixture. The mixture was stirred for $2 \mathrm{~h}$ at $0{ }^{\circ} \mathrm{C}$ in an ice water bath. The solvent was removed by a rotary evaporator at $50{ }^{\circ} \mathrm{C}$ and a white precipitate was formed. The white precipitate was then washed with diethyl ether and filtered using a Buchner filtration system. After filtration, the white powder was dissolved into hot ethanol to obtain a supersaturated solution. The solution was put into a freezer overnight. The white precipitate was then washed with diethyl ether and collected by vacuum filtration. After filtration, the large white crystallites (benzylammonium bromide, BenABr) were collected and dried in a vacuum oven at $60^{\circ} \mathrm{C}$ overnight. It was then transferred to an $\mathrm{N}_{2}$ glovebox where it was weighed ahead of device fabrication.

Material preparation: In this work, all materials were stored and prepared in $\mathrm{N}_{2}$ filled glovebox and were used as purchased without any additional purification unless otherwise stated. All solvents, unless otherwise stated, used in the work were purchased from Sigma Aldrich. To form the hole transport layer (HTL) solution, poly(4-butylphenyl-diphenyl-amine) (poly-TPD, 1-Material) was dissolved in toluene at a concentration of $1 \mathrm{mg} \cdot \mathrm{mL}^{-1}$ with $20 \mathrm{wt} \%$ 2,3,5,6-Tetrafluoro-7,7,8,8-tetracyanoquinodimethane ( $\mathrm{F}_{4}$-TCNQ, Lumtec). For the electron transport layer (ETL), [6,6]-phenyl-C61-butyric acid methyl ester ( $>99.5 \%$, PCBM, Solenne $\mathrm{BV}$ ) was prepared at a concentration of $20 \mathrm{mg} \cdot \mathrm{mL}^{-1}$ in a $3: 1$ (by volume) solution of chlorobenzene:dichlorobenzene. The hole blocking layer was prepared by dissolving bathocuproine (98\%, BCP, Alpha Aesar) at $0.5 \mathrm{mg} \cdot \mathrm{mL}^{-1}$ in isopropanol (IPA). This solution was heated at $100{ }^{\circ} \mathrm{C}$ and stirred overnight before deposition. To form the perovskite precursor solution, lead iodide (99.99 \%, $\mathrm{PbI}_{2}$, Alfa Aesar), lead bromide ( $>98 \%, \mathrm{PbBr}_{2}$, Alfa Aesar), cesium iodide (99.999 \%, CsI, Alfa Aesar), formamidinium iodide (FAI, GreatCell Solar) and methylammonium iodide (MAI, GreatCell Solar) were weighed to the appropriate stoichiometry to form the $\mathrm{Cs}_{0.05}\left(\mathrm{FA}_{0.83} \mathrm{MA}_{0.17}\right)_{0.95} \mathrm{~Pb}\left(\mathrm{I}_{0.90} \mathrm{Br}_{0.1}\right)_{3}$ (FAMACs) perovskite and then dissolved in a 4:1 ratio (by volume) of N,N-dimethylformamide (DMF) and dimethyl sulfoxide (DMSO) to achieve a concentration of $1.4 \mathrm{M}$. The BenABr salt was dissolved in chlorobenzene.

Device fabrication: Devices were fabricated in a planar device architecture glass/FTO/polyTPD/perovskite/PCBM/Ag. Fluorine-dope tin oxide (FTO) glass (Pilkington TEC 7, $7 \Omega \square^{-1}$ 
sheet resistivity) was etched with $\mathrm{Zn}$ powder and $2 \mathrm{M} \mathrm{HCl}$ acid to achieve the desired electrode pattern. The substrates were then successively cleaned by sonication in a diluted Hellmanex solution ( $1 \%$ vol in DI water) and deionized water for 10 minutes each before being rinsed in acetone and then isopropanol. Following this these substrates were treated with UV-ozone plasma for 15 minutes.

Immediately onto these substrates $70 \mu \mathrm{L}$ of $\mathrm{F}_{4} \mathrm{TCNQ}$ doped poly-TPD solution was deposited using the dynamic spincoating method in air (relative humidity $\sim 40 \%$ ) at $2000 \mathrm{rpm}$. After spinning for $20 \mathrm{~s}$, the films were annealed at $130{ }^{\circ} \mathrm{C}$ for 15 minutes. The substrates were then transferred to a $\mathrm{N}_{2}$ filled glovebox where the following spincoating procedures were carried out.

For the FAMACs perovskite, $170 \mu \mathrm{L}$ of precursor solution was deposited dynamically onto the substrate at $1000 \mathrm{rpm}$. After remaining at this speed for $9 \mathrm{~s}$, the speed was increased to 5000 rpm over $4 \mathrm{~s}$, where it remained for $45 \mathrm{~s}$. A solvent quench with $400 \mu \mathrm{L}$ of toluene $5 \mathrm{~s}$ before the end of the program was performed and the films were annealed at $100{ }^{\circ} \mathrm{C}$ for 1 hour. The films were left to cool before any additional layer was applied.

If present, the passivation layer was applied by dynamically spincoating $40 \mu \mathrm{L}$ of the BenABr solution at the desired concentration onto the perovskite film at $2000 \mathrm{rpm}$. After spinning for $20 \mathrm{~s}$, the films were annealed at $100^{\circ} \mathrm{C}$ for 1 minute.

PCBM was dynamically spin coated on top of the perovskite layer at $2000 \mathrm{rpm}$ using $40 \mu \mathrm{L}$ of solution and annealed for 3 minutes at $100{ }^{\circ} \mathrm{C}$. BCP was dynamically spin coated on top of the PCBM layer at $5000 \mathrm{rpm}$ using $100 \mu \mathrm{L}$ of solution with a 1 minute anneal at $100{ }^{\circ} \mathrm{C}$. Finally, metal electrodes were deposited by thermal evaporation. A Kurt J. Lesker Nano 36 evaporator was used to deposit $100 \mathrm{~nm}$ silver electrodes by thermal evaporation under high vacuum $\left(\sim 10^{-}\right.$ ${ }^{6}$ mbar) at a rate of approximately $0.05 \mathrm{~nm} \cdot \mathrm{s}^{-1}$. For the stability measurements, $3 \mathrm{~nm}$ of Cr was first deposited at a rate of $0.01 \mathrm{nms}^{-1}$, before $100 \mathrm{~nm}$ of Au at $\sim 0.02 \mathrm{nms}^{-1}$. The electrodes had an active area of $0.0919 \mathrm{~cm}^{2}$.

Device characterization: Current-voltage (JV) characteristics were recorded in ambient air under simulated AM1.5 solar light (1 sun, $100 \mathrm{mWcm}^{-2}$ ) generated by an ABET Class AAB sun 2000 simulator. Each device was measured with a $20 \mathrm{mV}$ voltage step and a $100 \mathrm{~ms}$ time step ( $\mathrm{scan}$ rate of $0.2 \mathrm{~V} \cdot \mathrm{s}^{-1}$ ). All devices were masked with a $0.0919 \mathrm{~cm}^{2}$ metal aperture to define the active area and eliminate edge effects. Stabilized power output (SPO) measurements were performed by holding the device at the voltage of the maximum power point, as determined by the JV characteristic, and monitoring the current density over the course of $60 \mathrm{~s}$. 
Device aging: Encapsulation was performed by affixing a glass cover slip to the complete devices using a UV-activated epoxy (LT-U001, Lumtec) in a $\mathrm{N}_{2}$ filled glovebox. The devices were then placed in an ATLAS Suntest XLS+ aging box where they were held at $85{ }^{\circ} \mathrm{C}$ and light soaked. The light source was a Xe arc lamp, filtered to closely match the AM1.5 solar spectrum. The light pulsed rapidly $(\sim 100 \mathrm{~Hz})$, which resulted in a time-average irradiance equivalent to 0.76 suns. Devices were removed 30 minutes prior to testing to allow them to return to room temperature.

Photoluminescence measurements: Time resolved PL measurements were recorded using a time correlated single photon counting (TCSPC) setup (FluoTime 300 PicoQuant GmbH). Photoluminescence quantum yield (PLQY) measurements were performed following the procedure of de Mello and co-workers. ${ }^{2}$ A $405 \mathrm{~nm}$ laser was used to photoexcite the samples placed in an integrating sphere and a Maya pro spectrometer used to measure the signal.

External quantum efficiency: The EQE was measured using a custom-built Fourier transform photocurrent spectrometer which is based on a Bruker Vertex 80v Fourier transform interferometer. The devices were illuminated with a $\sim 0.7$ sun $\left(70 \mathrm{mWcm}^{-2}\right)$, AM 1.5 spectrum generated by an Oriel class AAA solar simulator. The solar cells were masked with a metal aperture, with a defined active area of $0.0625 \mathrm{~cm}^{2}$. To calculate the EQE, the device photocurrent spectrum was divided by that of a calibrated silicon reference cell spectrum with a known EQE. The acquisition time for each spectrum was approximately $1 \mathrm{~min}$.

Terahertz spectroscopy: Terahertz time-domain spectroscopy (THz-TDS) and optical-pumpterahertz-probe spectroscopy (OPTPS) were used to investigate the conductivity and chargecarrier mobilities of the sample. The $\mathrm{THz}$ pulse was generated by a spintronic emitter and detected by a (110) ZnTe crystal using electro-optic sampling technique. The sample was photoexcited by a 400-nm wavelength 35 fs laser pulse at fluences ranging from 0.45 to 5.2 $\mu \mathrm{Jcm}^{-2}$, and the corresponding transient absorption spectra is shown in Figure S4.

$X$-ray diffraction: X-ray diffraction patterns were measured using a Panalytical X'pert powder diffractometer with a copper K $\alpha$ X-ray source, $\lambda=0.1541 \mathrm{~nm}$.

Scanning electron microscopy: Scanning Electron Microscopy images were taken using a FEI Quanta 600 FEG with an accelerating voltage of $5 \mathrm{kV}$.

X-ray photoemission spectroscopy: A Thermo Scientific Ka X-Ray Photoelectron spectrometer was used to perform XPS measurements using a monochromatic Al Ka X-Ray source at a take-off angle of 90 degrees. The core level XPS spectra were recorded using a pass energy of $20 \mathrm{eV}$ (resolution approximately $0.4 \mathrm{eV}$ ) from an analysis area of $300 \mu \mathrm{m} \times 300 \mu \mathrm{m}$. The spectrometer work function and binding energy scale were calibrated using the Fermi edge 
and $3 d$ peak recorded from a polycrystalline silver $(\mathrm{Ag})$ sample prior to the commencement of the experiments. Fitting procedures to extract peak positions from the XPS data were carried out using Casa XPS software suite. A Shirley background was used and the spectra were fit with a mixture of Gaussian/Lorentzian (Lorentzian $=30 \%)$ line shapes.

\section{Supporting note 1 - QFLS determination}

The quasi-Fermi level spitting (QFLS) was calculated according to previous reports. ${ }^{3,4}$ In this work, we calculate the QFLS according to

$$
Q F L S=Q F L S_{\text {rad }}+k_{\mathrm{B}} T \ln (P L Q Y),
$$

where $Q F L S_{\text {rad }}$ is the QFLS in the radiative limit, $k_{\mathrm{B}}$ is Boltzmann's constant and $T$ is temperature. Note that $P L Q Y$ in this equation is expressed in absolute terms (i.e. not as a percentage). We can then calculate the boost in $Q F L S$ owing to the passivation treatment by evaluating $\triangle Q F L S=Q F L S_{\text {passivated }}-Q F L S_{\text {pristine. Assuming that the passivation does not }}$ change the bandgap, and so the QFLS in the radiative limit is constant (1.31 eV in our case), we can simplify the expression to

$$
\Delta \mathrm{QFLS}=k_{\mathrm{B}} T \ln \left(\frac{P L Q Y_{\text {passivated }}}{P L Q Y_{\text {pristine }}}\right)=25.7 \mathrm{meV} \times \ln \left(\frac{P L Q Y_{\text {passivated }}}{P L Q Y_{\text {pristine }}}\right),
$$

Where we have substituted for room temperature $(298 \mathrm{~K})$ for the final expression. This expression provides a straightforward way to calculate the expected gain in open-circuit voltage from a passivation treatment.

\section{Supporting Note 2 - TRPL fitting}

Time resolved photoluminescence decays were fitted using a stretched exponential,

$$
I(t)=A e^{-\left(\left(t-t_{0}\right) / \tau\right)^{\beta}}+C,(\mathrm{~S} 3)
$$

where $A$ is a normalizing constant, $t$ is time, $t_{0}$ an initial time offset, $C$ is a constant offset in intensity, $\tau$ is an analogue to lifetime and $\beta$ is the stretching factor. The mean relaxation time, which we define as the lifetime, is the area under the decay curves and hence is calculated as

$$
\langle\tau\rangle=\int_{0}^{\infty} A e^{-(t / \tau)^{\beta}} d t=A \frac{\tau}{\beta} \Gamma\left(\frac{1}{\beta}\right),
$$

where $\Gamma$ is the gamma function. Applying a least squares regression to the decays (between 5 and $2500 \mathrm{~ns}$ ) presented in Figure 1b, we obtain the following parameters

\begin{tabular}{|l|l|l|}
\hline \multicolumn{1}{|c|}{ Parameter } & \multicolumn{1}{|c|}{ Control } & \multicolumn{1}{c|}{ BenABr } \\
\hline$A$ & 0.74 & 0.93 \\
\hline$\tau(\mathrm{ns})$ & 95 & 315 \\
\hline
\end{tabular}




\begin{tabular}{|l|l|l|}
\hline$\beta$ & 0.73 & 0.67 \\
\hline$t_{0}(\mathrm{~ns})$ & 20 & 6.4 \\
\hline$C$ & $3.9 \times 10^{-3}$ & $1.7 \times 10^{-2}$ \\
\hline$\langle\tau\rangle(\mathrm{ns})$ & 85 & 394 \\
\hline
\end{tabular}

\section{Supporting Note 3 - Absorption coefficient calculation}

We calculate the absorption coefficient of the perovskite films from the terahertz spectroscopic studies as follows. We first obtain the complex conductivity as a function of frequency:

$$
\sigma(\omega)=\sigma_{\mathrm{re}}(\omega)+i \sigma_{\mathrm{im}}(\omega)
$$

where $i$ is the square root of -1 and $\sigma_{\text {re/im }}$ refers to the real/imaginary part of the dark conductivity in Figure $\mathbf{S 2}$ and $\omega$ is frequency. We then calculate the dielectric function according to

$$
\varepsilon_{r}(\omega)=\varepsilon_{\infty}+\frac{i}{\varepsilon_{0} \omega} \sigma(\omega)
$$

where $\varepsilon_{\infty}$ is the dielectic function at high frequency and is assumed to be $30 \mathrm{Fm}^{-1}, \varepsilon_{0}$ is the vacuum permittivity. The real and imaginary parts, $n$ and $k$ respectively, of the refractive index can be obtained by taking the square root of the dielectric function. Then the absorption coefficient is calculated using

$$
\alpha(\omega)=\frac{4 \pi \omega}{c k}
$$

where $c$ is the speed of light.

\section{Supporting note 4 - Drude fit}

Using optical-pump-terahertz-probe spectroscopy (OPTPS), we measured the photoconductivity spectrum $(\Delta \sigma)$ of the samples under photoexcitation fluence of $2 \mu \mathrm{Jcm}^{-2}$. According to the following equation, the photoconductivity can be expressed in terms of $\mathrm{THz}$ transitivity: 


$$
\Delta \sigma(\omega)=-\frac{\varepsilon_{0} c\left(1+n_{S}\right)}{d} \frac{\Delta T(\omega)}{T(\omega)}, \quad(\mathrm{S} 8)
$$

where $\varepsilon_{0}$ is the permittivity of free space, $n_{\mathrm{s}}$ is the refractive index of the quartz substrate and $d$ is the thickness of the thin film. $\Delta T$ is the difference in $\mathrm{THz}$ transmission between photoexcitation and non-photoexcitation. As shown in Figure S4, the photoconductivity spectrum shows a Drude response with a relatively flat real part (blue dots) and negligible imaginary part (red dots), on top of which phonon modulation occurs at $1 \mathrm{THz}$ and $2 \mathrm{THz}$, due to electron-phonon coupling. The Drude model is expressed analytically by the following equation:

$$
\Delta \sigma(\omega)=\frac{N e^{2}}{m} \frac{i}{\omega+i \gamma}
$$

where $e$ is the electron charge, $N$ is the electron density and $m$ is the electron effective mass. $\gamma$ is the momentum scattering rate, from which the electron mobility can be extracted:

$$
\mu=\frac{e}{m \gamma}
$$

By fitting the above Drude model to the measured photoconductivity spectrum, the mobility of the control and treated films are extracted to be $(51.2 \pm 3.7)$ and $(62.5 \pm 4.2) \mathrm{cm}^{2} \mathrm{~V}^{-1} \mathrm{~s}^{-1}$, respectively.

\section{Supporting note 5 - Diffusion length Calculation}

The diffusion length can be calculated according to

$$
L_{\mathrm{d}}=\sqrt{\frac{D}{R_{\mathrm{tot}}(n)}}=\sqrt{\frac{\mu k_{\mathrm{B}} T}{e R_{\mathrm{tot}}(n)}},
$$

where we have substituted the diffusion coefficient, $D$, for $\mu k_{\mathrm{B}} T / e$, and $k_{\mathrm{B}} T$ is the thermal energy and $e$ the elementary charge. ${ }^{5,6} R_{\text {tot }}$ is the total charge-carrier recombination rate and is given by $R_{\text {tot }}(n)=n k_{2}+k_{1}$, where $k_{1}$ and $k_{2}$ and the trap-mediated and bimolecular recombination rates respectively. We obtain $k_{1}$ from fitting the photoluminescence decays in Figure 1a, yielding values of $k_{1}=3.1 \times 10^{7} \mathrm{~s}^{-1}$ and $k_{1}=4.1 \times 10^{6} \mathrm{~s}^{-1}$ for pristine and treated films respectively. In principle, these $k_{1}$ values can be used as fixed parameters to fit the $\mathrm{THz}$ transient decays for $k_{2}$, shown in Figure S4, using the method outlined in previous reports. ${ }^{4,5}$ However, at charge- 
carrier densities typical at AM1.5, $n=10^{14} \mathrm{~cm}^{-3}$, the effect of bimolecular recombination is small, since $k_{1} \gg n k_{2}$, and so the values of $k_{2}$ have a negligible effect on the calculated diffusion lengths. 


\section{Supporting Note 6 - Charge carrier transport discussion}

The short-circuit current density can be increased by improving the generation efficiency, the collection efficiency of charge carriers, or both. Our measurements probe the charge-carrier transport properties which will impact the collection efficiency. A consensus is emerging in the field that it is the diffusion current that dominates charge transport in PSCs (given sufficiently selective charge transport layers), however recent has shown that a built-in potential is required for optimal device performance. ${ }^{7,8}$ This built-in potential results in a drift current which helps to extract carriers at $0 \mathrm{~V}$.

In our work, the improvements in charge carrier lifetimes, mobilities and diffusion lengths indicate a higher quality charge-carrier transport within the perovskite film in the presence of the BenABr passivation treatment. The electric field from the $\mathrm{THz}$ probe during the OPTPS measurements, induces and probes drift current to determine charge-carrier mobility. As such, combining these measurements with the recombination parameters from OPTPS and TRPL gives an accurate indication of the charge carrier transport within the perovskite semiconductor. ${ }^{9}$ Therefore, given that we see improved charge-carrier transport in the treated films, we can have confidence that the charge collection efficiency will be increased. This is verified by the panchromatic increase in the external quantum efficiency of the devices. However, we note that this is unlikely to be the full picture. Gaining precise understanding about improvements to the short circuit current density following passivation requires significant further studies which are currently being undertaken.

\section{References}

1. Wagner, C. D.; Riggs, W. M.; Davis, L. E.; Moulder, J. F.; Muilenberg, G. E.

Handbook of X-Ray Photoelectron Spectroscopy; Perkin-Elmer Corporation: 1979.

2. de Mello, J.C.; Wittmann, H.F.; Friend, R.H., An improved experimental determination of external photoluminescence quantum efficiency. Adv. Mater., 1997 9: 230232. doi:10.1002/adma.19970090308

3. Stolterfoht, M.; Caprioglio, P.; Wolff, C. M.; Márquez, J. A.; Nordmann, J.; Zhang, S.; Rothhardt, D.; Hörmann, U.; Amir, Y.; Redinger, A.; Kegelmann, L.; Zu, F.; Albrecht, S.; Koch, N.; Kirchartz, T.; Saliba, M.; Unold, T.; Neher, D. The Impact of Energy Alignment and Interfacial Recombination on the Internal and External Open-Circuit Voltage of 
Perovskite Solar Cells. Energy Environ. Sci. 2019, 12 (9), 2778-2788.

https://doi.org/10.1039/c9ee02020a.

4. Caprioglio, P.; Stolterfoht, M.; Wolff, C. M.; Unold, T.; Rech, B.; Albrecht, S.; Neher, D. On the Relation between the Open-Circuit Voltage and Quasi-Fermi Level Splitting in Efficient Perovskite Solar Cells. Adv. Energy Mater. 2019, 9 (33). https://doi.org/10.1002/aenm.201901631.

5. Milot, R. L.; Eperon, G. E.; Snaith, H. J.; Johnston, M. B.; Herz, L. M. TemperatureDependent Charge-Carrier Dynamics in CH3NH3PbI3 Perovskite Thin Films. Adv. Funct. Mater. 2015, 25 (39), 6218-6227. https://doi.org/10.1002/adfm.201502340.

6. $\quad$ Buizza, L. R. V.; Crothers, T. W.; Wang, Z.; Patel, J. B.; Milot, R. L.; Snaith, H. J.; Johnston, M. B.; Herz, L. M. Charge-Carrier Dynamics, Mobilities, and Diffusion Lengths of 2D-3D Hybrid Butylammonium-Cesium-Formamidinium Lead Halide Perovskites. $A d v$. Funct. Mater. 2019, 29 (35). https://doi.org/10.1002/adfm.201902656.

7. Sandberg, O. J.; Kurpiers, J.; Stolterfoht, M.; Neher, D.; Meredith, P.; Shoaee, S.; Armin, A. On the Question of the Need for a Built-In Potential in Perovskite Solar Cells. Adv. Mater. Interfaces 2020, 7 (10). https://doi.org/10.1002/admi.202000041.

8. Kirchartz, T.; Bisquert, J.; Mora-Sero, I.; Garcia-Belmonte, G. Classification of Solar Cells According to Mechanisms of Charge Separation and Charge Collection. Phys. Chem. Chem. Phys. 2015, 17 (6), 4007-4014. https://doi.org/10.1039/c4cp05174b.

9. Johnston, M. B.; Herz, L. M. Hybrid Perovskites for Photovoltaics: Charge-Carrier Recombination, Diffusion, and Radiative Efficiencies. Acc. Chem. Res. 2016, 49 (1), 146-154. https://doi.org/10.1021/acs.accounts.5b00411. 